\title{
Ultradifferentiable Fundamental Kernels of Linear Partial Differential Operators on Non-quasianalytic Classes of Roumieu Type
}

\author{
By
}

Angela A. Albanese* and José Bonet**

\begin{abstract}
Let $P$ be a linear partial differential operator with coefficients in the Roumieu class $\mathcal{E}_{\{\omega\}}(\Omega)$. We prove that if $P$ and its transposed operator ${ }^{t} P$ are $\{\omega\}$-hypoelliptic in $\Omega$ and surjective on the space $\mathcal{E}_{\{\omega\}}(\Omega)$, then $P$ has a global two-sided ultradifferentiable fundamental kernel in $\Omega$, thus extending to the Roumieu classes the well-known analogous result of B. Malgrange in the $C^{\infty}$ class. This result is new even for Gevrey classes.
\end{abstract}

\section{$\S 1 . \quad$ Introduction and Preliminaries}

Ehrenpreis [5] and Malgrange [17] proved that every linear partial differential operator with constant coefficients has a fundamental solution; therefore the inhomogeneous equation $P u=f$ admits always $C^{\infty} / \mathcal{E}_{\{\omega\}}$-solutions for each right hand side $f$ in the class $C^{\infty} / \mathcal{E}_{\{\omega\}}$ respectively, and with compact support. However, it is well-known that this is not longer the case for linear partial differential operators with variable coefficients, i.e., see the Lewy's operator. The

Communicated by T. Kawai. Received August 3, 2005. Revised November 15, 2005.

2000 Mathematics Subject Classification(s): 35H10, 46F05, 46E40, 47B38.

Key words: Fundamental kernel, ultradifferentiable function spaces of Roumieu type, ultradistribution spaces of Roumieu type, hypoelliptic operators.

The present research was partially supported by the Integrated Action between Italy and Spain HI2003-66. J. Bonet was also partially supported by MEC and FEDER Project MTM2004-02262, AVCIT Grupos 03/050 and the net MTM2004-21420-E.

*Dipartimento di Matematica "E. De Giorgi", Università di Lecce, I-73100 Lecce, Italy. e-mail: angela.albanese@unile.it

** Dipartamento de Matemática, E.T.S. Arquitectura, Universidad Politécnica de Valencia, E-46071 Valencia, Spain.

e-mail: jbonet@mat.upv.es

(C) 2007 Research Institute for Mathematical Sciences, Kyoto University. All rights reserved. 
condition of hypoellipticity of the operator $P$ on an open set $\Omega$ ensures that its transposed operator ${ }^{t} P$ is locally solvable, as it was proved in [7] in the $C^{\infty}$ case and in [2] in the frame of Gevrey classes. On the other hand, under the assumptions that $P$ and ${ }^{t} P$ are both hypoelliptic in an open set $\Omega$ and surjective on $C^{\infty}(\Omega)$, Malgrange [17] showed that $P$ has a two-sided fundamental kernel, thereby obtaining that the inhomogeneous equation $P u=f$ admits a linear continuous solution operator on the space of $C^{\infty}$ functions with compact support.

The purpose of this paper is to extend the classical result of Malgrange [17] to the setting of ultradifferentiable function spaces $\mathcal{E}_{\{\omega\}}$ of Roumieu type, in particular for Gevrey classes (see Theorem 2.2). The topological structure of these spaces is much more involved than that of Fréchet spaces of $C^{\infty}$ functions. The main result is based on Theorem 2.1 about the existence of local two-sided kernels, which extends (and improves) the work of Morando [20] from Gevrey classes to the case of general non-quasianalytic classes as defined by Braun, Meise and Taylor [3]; see the details below. Our results are obtained as an application of topological tensor products and spaces of vector valued ultradistributions and ultradifferentiable functions to linear partial differential operators. Several authors, like Gramchev or Rodino, had asked the authors about the validity of Malgrange result for Gevrey classes. We believe that it might have useful consequences; compare with [1]. This research continues work of A. Corli, L. Rodino, A. Morando and the first author. In particular, certain estimates from [2] are essential. The paper concludes with a few examples of partial differential operators with variable coefficients to which our results can be applied.

We refer the reader to $[10,14,24]$ for functional analysis, to $[8,9]$ for the theory of linear partial differential operators, and $[6,10,14,24]$ for properties of topological tensor products. We fix notation and give some definitions and results which will be useful for the sequel. Following Braun, Meise and Taylor [3], we introduce the classes of non-quasianalytic functions of Roumieu type.

Definition 1.1. A continuous increasing function $\omega:[0, \infty[\rightarrow[0, \infty[$ is called a weight function if it has the following properties:

$(\alpha)$ there exists $K \geq 1$ with $\omega(2 t) \leq K(1+\omega(t))$ for all $t \geq 0$,

( $\beta) \int_{0}^{\infty} \frac{\omega(t)}{1+t^{2}} d t<\infty$,

$(\gamma) \log t=o(\omega(t))$ for $t \rightarrow \infty$ 
( $\delta) \varphi: t \rightarrow \omega\left(e^{t}\right)$ is convex on $\mathbb{R}$.

For a weight function $\omega$ we define $\tilde{\omega}: \mathbb{C}^{N} \rightarrow[0, \infty[$ by $\tilde{\omega}(z):=\omega(|z|)$ and again denote this function by $\omega$. The Young conjugate $\varphi^{*}:[0, \infty[\rightarrow \mathbb{R}$ of $\varphi$ is defined by

$$
\varphi^{*}(y):=\sup _{x \geq 0}(x y-\varphi(x))
$$

Example 1. The following functions $\omega:[0, \infty[\rightarrow[0, \infty[$ are examples of weight functions: $\omega(t)=t^{\alpha}, 0<\alpha<1$; $\omega(t)=(\log (1+t))^{\beta}, \beta>1$; $\left.\omega(t)=\exp \left(\beta(\log (1+t))^{\alpha}\right)\right), 0<\alpha<1, \beta>0 ; \omega(t)=t(\log (e+t))^{-\beta}, \beta>1$.

We point out that for $\omega(t)=t^{\alpha}$ the classes of functions defined below coincide with the Gevrey class $G^{s}$ for $s=1 / \alpha$. This is the most important example we have in mind.

Definition 1.2. Let $\omega$ be a weight function.

(a) For a compact set $K$ in $\mathbb{R}^{N}$ which coincides with the closure of its interior and $\mu>0$ let

$$
\begin{aligned}
& \mathcal{E}_{\omega}(K, \mu) \\
& :=\left\{f \in C^{\infty}(K):\|f\|_{K, \mu}:=\sup _{x \in K} \sup _{\alpha \in \mathbb{N}_{0}^{N}}\left|f^{(\alpha)}(x)\right| \exp \left(-\mu \varphi^{*}(|\alpha| / \mu)\right)<\infty\right\}
\end{aligned}
$$

which is a Banach space endowed with the \|\|$_{K, \mu}$-topology.

(b) For a compact set $K$ in $\mathbb{R}^{N}$ which coincides with the closure of its interior let

$$
\mathcal{E}_{\{\omega\}}(K):=\operatorname{ind}_{m \rightarrow \infty} \mathcal{E}_{\omega}(K, 1 / m)
$$

which is the strong dual of a nuclear Fréchet space (i.e., a (DFN)-space) if it is endowed with its natural inductive limit topology [3].

(c) For an open set $\Omega$ in $\mathbb{R}^{N}$ we define

$$
\mathcal{E}_{\{\omega\}}(\Omega):=\operatorname{proj}_{K \subset \subset \Omega} \mathcal{E}_{\{\omega\}}(K)
$$

and we endow $\mathcal{E}_{\{\omega\}}(\Omega)$ with its natural projective topology. The elements of $\mathcal{E}_{\{\omega\}}(\Omega)$ are called $\omega$-ultradifferentiable functions of Roumieu type on $\Omega$. By [3, Proposition 4.9] $\mathcal{E}_{\{\omega\}}(\Omega)$ is a complete, nuclear and reflexive locally convex space. In particular, $\mathcal{E}_{\{\omega\}}(\Omega)$ is also an ultrabornological (hence barrelled and bornological) space as it follows from [25] (or see [26, Theorem 3.3.4]) and [4, Lemma 1.8]). We denote by $\mathcal{E}_{\{\omega\}}^{\prime}(\Omega)$ the strong dual of $\mathcal{E}_{\{\omega\}}(\Omega)$. 
(d) For a compact set $K$ in $\mathbb{R}^{N}$ which coincides with the closure of its interior let

$$
\mathcal{D}_{\{\omega\}}(K):=\left\{f \in \mathcal{E}_{\{\omega\}}\left(\mathbb{R}^{N}\right): \operatorname{supp} f \subset K\right\},
$$

endowed with the induced topology. In [3, Remark 3.2 (1) and Corollary 3.6 (1)] it is shown that $\mathcal{D}_{\{\omega\}}(K) \neq\{0\}$ is the strong dual of a Fréchet nuclear space (i.e., (DFN)-space). For an open set $\Omega$ in $\mathbb{R}^{N}$ let

$$
\mathcal{D}_{\{\omega\}}(\Omega):=\operatorname{ind}_{K \subset \subset \Omega} \mathcal{D}_{\{\omega\}}(K) .
$$

The elements of its strong dual $\mathcal{D}_{\{\omega\}}^{\prime}(\Omega)$ are called $\omega$-ultradistributions of Roumieu type on $\Omega$.

Let $S_{\omega}:=\{\sigma: \sigma$ is a weight function satisfying $\sigma=o(\omega)\}$. By [19, Corollary 3.3$]$ the family of seminorms defined by

$$
\|f\|_{K, \sigma}:=\sup _{x \in K} \sup _{\alpha \in \mathbb{N}_{0}^{N}}\left|f^{(\alpha)}(x)\right| \exp \left(-\varphi_{\sigma}^{*}(|\alpha|)\right), \quad f \in \mathcal{E}_{\{\omega\}}(\Omega),
$$

where $K \subset \subset \Omega, \sigma \in S_{\omega}$, is a fundamental system of continuous seminorms on $\mathcal{E}_{\{\omega\}}(\Omega)$ and hence on $\mathcal{D}_{\{\omega\}}(K)$ for all compact subset $K$ of $\Omega$. From [3, Lemma 3.3 and Proposition 3.4] it follows that the family of seminorms

$$
\|f\|_{2, \sigma}:=\left(\int_{\mathbb{R}^{N}}|\hat{f}(t)|^{2} e^{2 \sigma(t)} d t\right)^{1 / 2}, \quad \sigma \in S_{\omega},
$$

is also a fundamental system of continuous seminorms on $\mathcal{D}_{\{\omega\}}(K)$.

For each $\sigma \in S_{\omega}$ we now introduce the space

$$
H_{\omega}^{\sigma}:=\left\{u \in \mathcal{S}^{\prime}\left(\mathbb{R}^{N}\right): \hat{u}(t) e^{\sigma(t)} \in L^{2}\left(\mathbb{R}^{N}\right)\right\} .
$$

It is easy to show that $H_{\omega}^{\sigma}$ is a Hilbert space with respect to the inner product defined by

$$
\langle u, v\rangle_{\sigma, \omega}:=\int_{\mathbb{R}^{N}} \hat{u}(t) \overline{\hat{v}}(t) e^{2 \sigma(t)} d t, \quad u, v \in H_{\omega}^{\sigma},
$$

and the following inclusions

$$
\mathcal{D}_{\{\omega\}}(K) \hookrightarrow H_{\omega}^{\sigma} \hookrightarrow L^{2}\left(\mathbb{R}^{N}\right)
$$

are continuous with dense ranges. Actually, $\left(\mathcal{D}_{\{\omega\}}(K),\|\|_{2, \sigma}\right)$ is continuously included in $L^{2}\left(\mathbb{R}^{N}\right)$ too, as

$$
\|f\|_{2}=(2 \pi)^{-N}\|\hat{f}\|_{2} \leq(2 \pi)^{-N}\|f\|_{2, \sigma}
$$


for every $f \in \mathcal{D}_{\{\omega\}}(K)$. Denoting by $H_{\{\omega\}}^{\sigma}(K)$ the closure of $\mathcal{D}_{\{\omega\}}(K)$ in $H_{\omega}^{\sigma}$, we have that $\left(H_{\{\omega\}}^{\sigma}(K),\|\|_{2, \sigma}\right)$ is a Hilbert space and its strong dual $\left(H_{\{\omega\}}^{\sigma}(K)\right)^{\prime}$ may be continuously embedded in $\mathcal{D}_{\{\omega\}}^{\prime}(K)$ with dense image. In particular, by (1.1) we have

$$
H_{\{\omega\}}^{\sigma}(K) \hookrightarrow L^{2}\left(\mathbb{R}^{N}\right) \text { continuously with dense range. }
$$

Vector-valued ultradifferentiable function and ultradistribution spaces of Roumieu type are defined as it follows. Let $F$ be a locally convex space. A vector-valued function $f: \Omega \rightarrow F$ is said to be an ultradifferentiable function of Roumieu type if it is infinitely differentiable as an $F$-valued function [10], see also [22, 23], and if for each continuous seminorm $q$ on $F$ and each compact set $K \subset \Omega$ there exists $m \in \mathbb{N}$ such that

$$
\sup _{x \in K} \sup _{\alpha \in \mathbb{N}_{0}^{N}} q\left(f^{(\alpha)}(x)\right) \exp \left(-\frac{1}{m} \varphi^{*}(m|\alpha|)\right)<\infty .
$$

We denote by $\mathcal{E}_{\{\omega\}}(\Omega, F)$ the space of all $F$-valued ultradifferentiable functions of Roumieu type on $\Omega$ endowed with the locally convex topology defined by the following family of seminorms

$$
\|f\|_{q, K, \sigma}:=\sup _{x \in K} \sup _{\alpha \in \mathbb{N}_{0}^{N}} q\left(f^{(\alpha)}(x)\right) \exp \left(-\varphi_{\sigma}^{*}(|\alpha|)\right),
$$

where $q \in \operatorname{cs}(F), K \subset \Omega$ is a compact set, $\sigma \in S_{\omega}$. If $F$ is complete, by [13, Theorem 3.10] a function $f: \Omega \rightarrow F$ belongs to $\mathcal{E}_{\{\omega\}}(\Omega, F)$ if and only if $u \circ f \in \mathcal{E}_{\{\omega\}}(\Omega)$ for all $u \in F^{\prime}$ and we have the following isomorphism

$$
\mathcal{E}_{\{\omega\}}(\Omega, F) \simeq \mathcal{E}_{\{\omega\}}(\Omega) \hat{\otimes}_{\pi} F \simeq L_{\varepsilon}\left(F_{\tau}^{\prime}, \mathcal{E}_{\{\omega\}}(\Omega)\right),
$$

where $\varepsilon$ denotes the topology of uniform convergence on the equicontinuous sets in $F^{\prime}$ and $F_{\tau}^{\prime}$ denotes the topological dual of $F$ equipped with the Mackey topology.

Similarly to Komatsu [13] we define the space of vector-valued ultradistributions of Roumieu type on $\Omega$ as

$$
\mathcal{D}_{\{\omega\}}^{\prime}(\Omega, F):=L_{\beta}\left(\mathcal{D}_{\{\omega\}}(\Omega), F\right),
$$

where $\beta$ denotes the topology of uniform convergence on the bounded sets in $\mathcal{D}_{\{\omega\}}(\Omega)$. An element $u \in \mathcal{D}_{\{\omega\}}^{\prime}(\Omega, F)$ is said to be an ultradistribution of Roumieu type on $\Omega$. If $F$ is complete, then we have the following canonical isomorphism

$$
\mathcal{D}_{\{\omega\}}^{\prime}(\Omega, F) \simeq \mathcal{D}_{\{\omega\}}^{\prime}(\Omega) \hat{\otimes}_{\pi} F \simeq L_{\varepsilon}\left(F_{\tau}^{\prime}, \mathcal{D}_{\{\omega\}}^{\prime}(\Omega)\right) .
$$




\section{§2. $\{\omega\}$-ultradifferentiable Fundamental Kernels of Hypoelliptic Partial Differential Operators}

\section{§2.1. $\{\omega\}$-ultradifferentiable fundamental kernels}

The theory of kernels of L. Schwartz can be extended to the setting of the classes of $\{\omega\}$-ultradifferentiable functions and $\{\omega\}$-ultradistributions. Indeed, using well known results of Grothendieck [6] on topological tensor products one can prove the following results (see, e.g., $[12,13,3]$ ).

Let $\Omega \subset \mathbb{R}^{N}, \Omega^{\prime} \subset \mathbb{R}^{M}$ be open sets. The bilinear map which assigns to each pair of functions $\varphi(x)$ on $\Omega$ and $\psi(y)$ on $\Omega^{\prime}$ the product $\varphi(x) \psi(y)$ on $\Omega \times \Omega^{\prime}$ induces the following canonical isomorphisms

$$
\begin{gathered}
\mathcal{E}_{\{\omega\}}(\Omega) \hat{\otimes}_{\pi} \mathcal{E}_{\{\omega\}}\left(\Omega^{\prime}\right) \simeq \mathcal{E}_{\{\omega\}}\left(\Omega \times \Omega^{\prime}\right) \\
\mathcal{D}_{\{\omega\}}(\Omega) \hat{\otimes}_{\pi} \mathcal{D}_{\{\omega\}}\left(\Omega^{\prime}\right) \simeq \mathcal{D}_{\{\omega\}}\left(\Omega \times \Omega^{\prime}\right)
\end{gathered}
$$

(see [12, Theorem 2.1]). Moreover, compare with [6, Théorème, Chap. II]

$$
\mathcal{D}_{\{\omega\}}^{\prime}(\Omega) \hat{\otimes}_{\pi} \mathcal{D}_{\{\omega\}}^{\prime}\left(\Omega^{\prime}\right) \simeq \mathcal{D}_{\{\omega\}}^{\prime}\left(\Omega \times \Omega^{\prime}\right)
$$

(see [12, Theorem 2.3]). We have also the following canonical isomorphism

$$
\mathcal{D}_{\{\omega\}}^{\prime}\left(\Omega \times \Omega^{\prime}\right) \simeq L_{\beta}\left(\mathcal{D}_{\{\omega\}}\left(\Omega^{\prime}\right), \mathcal{D}_{\{\omega\}}^{\prime}(\Omega)\right) \simeq L_{\beta}\left(\mathcal{D}_{\{\omega\}}(\Omega), \mathcal{D}_{\{\omega\}}^{\prime}\left(\Omega^{\prime}\right)\right)
$$

which maps every $\{\omega\}$-ultradistribution $K(x, y)$ on $\Omega \times \Omega^{\prime}$ onto the linear continuous map $K: \mathcal{D}_{\{\omega\}}\left(\Omega^{\prime}\right) \rightarrow \mathcal{D}_{\{\omega\}}^{\prime}(\Omega)$ defined by

$$
\langle K(\psi), \varphi\rangle:=\langle K, \varphi \otimes \psi\rangle
$$

for all $\varphi \in \mathcal{D}_{\{\omega\}}(\Omega), \quad \psi \in \mathcal{D}_{\{\omega\}}\left(\Omega^{\prime}\right)$, where $(\varphi \otimes \psi)(x, y):=\varphi(x) \psi(y)$ for all $x \in \Omega, y \in \Omega^{\prime}$ (see [12, Theorem 2.3]). Formally, we can write

$$
(K \psi)(x)=\int_{\Omega^{\prime}} K(x, y) \psi(y) d y .
$$

Under the correspondence (2.5) the following isomorphism holds

$$
\mathcal{E}_{\{\omega\}}\left(\Omega \times \Omega^{\prime}\right) \simeq L_{\beta}\left(\mathcal{E}_{\{\omega\}}^{\prime}\left(\Omega^{\prime}\right), \mathcal{E}_{\{\omega\}}(\Omega)\right)
$$

Following Malgrange [17] (see also [13, 21]) we introduce the following properties. 
Definition 2.1. Let $\Omega \subset \mathbb{R}^{N}, \Omega^{\prime} \subset \mathbb{R}^{M}$ be open sets and let $K(x, y) \in$ $\mathcal{D}_{\{\omega\}}^{\prime}\left(\Omega \times \Omega^{\prime}\right)$. The kernel $K$ is said to be $\{\omega\}$-semiregular in $x$ if $(2.5)$ defines a linear continuous map from $\mathcal{D}_{\{\omega\}}\left(\Omega^{\prime}\right)$ to $\mathcal{E}_{\{\omega\}}(\Omega)$; while the kernel $K$ is said to be $\{\omega\}$-semiregular in $y$ if the corresponding map $K$ defined in (2.5) can be extended as a linear continuous map from $\mathcal{E}_{\{\omega\}}^{\prime}\left(\Omega^{\prime}\right)$ into $\mathcal{D}_{\{\omega\}}^{\prime}(\Omega)$ or equivalently, the transposed map ${ }^{t} K$ is semiregular in $x$. We say that the kernel $K$ is $\{\omega\}$-regular if it is $\{\omega\}$-semiregular in $x$ and $y$. The kernel $K$ is said to be $\{\omega\}$-regularizing if it is an element of $\mathcal{E}_{\{\omega\}}\left(\Omega \times \Omega^{\prime}\right) \simeq \mathcal{E}_{\{\omega\}}(\Omega) \hat{\otimes}_{\pi} \mathcal{E}_{\{\omega\}}\left(\Omega^{\prime}\right)$ or equivalently, see (2.7), the corresponding map $K$ can be extended to a linear continuous map from $\mathcal{E}_{\{\omega\}}^{\prime}\left(\Omega^{\prime}\right)$ into $\mathcal{E}_{\{\omega\}}(\Omega)$. Finally, a kernel $K \in \mathcal{D}_{\{\omega\}}^{\prime}(\Omega \times \Omega)$ is said to be $\{\omega\}$-very regular if it is $\{\omega\}$-regular and it is also a $\mathcal{E}_{\{\omega\}}$-function in the complement of the diagonal $\Delta$ in $\Omega \times \Omega$.

Remark. Via the following isomorphisms

$$
\begin{aligned}
& L_{\beta}\left(\mathcal{D}_{\{\omega\}}\left(\Omega^{\prime}\right), \mathcal{E}_{\{\omega\}}(\Omega)\right) \simeq \mathcal{E}_{\{\omega\}}\left(\Omega, \mathcal{D}_{\{\omega\}}^{\prime}\left(\Omega^{\prime}\right)\right), \\
& L_{\beta}\left(\mathcal{E}_{\{\omega\}}^{\prime}\left(\Omega^{\prime}\right), \mathcal{D}_{\{\omega\}}^{\prime}(\Omega)\right) \simeq \mathcal{E}_{\{\omega\}}\left(\Omega^{\prime}, \mathcal{D}_{\{\omega\}}^{\prime}(\Omega)\right)
\end{aligned}
$$

(compare with [13, Theorem 5.2]), the kernels which are $\{\omega\}$-semiregular in $x \in \Omega$ can be identified with the $\mathcal{E}_{\{\omega\}}$-functions of $x \in \Omega$ valued in $\mathcal{D}_{\{\omega\}}^{\prime}\left(\Omega^{\prime}\right)$ with respect to $y$, while the kernels which are $\{\omega\}$-semiregular in $y$ can be identified with the $\mathcal{E}_{\{\omega\}}$-functions of $y \in \Omega^{\prime}$ valued in $\mathcal{D}_{\{\omega\}}^{\prime}(\Omega)$ with respect to $x$.

\section{§2.2. $\{\omega\}$-hypoelliptic partial differential operators}

Let

$$
P=P(x, D)=\sum_{|\alpha| \leq m} a_{\alpha} D^{\alpha}
$$

be a partial differential operator, where $D^{\alpha}=D_{1}^{\alpha_{1}} \ldots D_{N}^{\alpha_{N}}, D_{j}=-i \partial / \partial x_{j}$, $|\alpha|=\alpha_{1}+\ldots+\alpha_{N}$ for any multi-index $\alpha \in \mathbb{N}_{0}^{N}$ and $m \in \mathbb{N}$. Suppose that the coefficients $a_{\alpha}$ of $P$ belong to $\mathcal{E}_{\{\omega\}}(\Omega), \Omega$ an open set of $\mathbb{R}^{N}$. Then $P$ continuously maps each of the spaces $\mathcal{E}_{\{\omega\}}(H), \mathcal{E}_{\{\omega\}}^{\prime}(H), \mathcal{D}_{\{\omega\}}(H)$ and $\mathcal{D}_{\{\omega\}}^{\prime}(H)$ into itself for every open or compact subset $H \subset \Omega$. We denote by $N(\omega, P, \Omega)$ (by $N(\omega, P, H)$, with $H \subset \Omega$, respectively) the kernel of $P$ acting on $\mathcal{E}_{\{\omega\}}(\Omega)$ (on $\mathcal{E}_{\{\omega\}}(H)$ respectively). The transposed operator ${ }^{t} P$ of $P$ is defined by $\langle P \varphi, \psi\rangle=$ $\left\langle\varphi,{ }^{t} P \psi\right\rangle$ for all $\varphi, \psi \in \mathcal{D}_{\{\omega\}}(\Omega)$, where $\langle\varphi, \psi\rangle:=\int_{\Omega} \varphi(x) \psi(x) d x$.

The operator $P$ is said to be $\{\omega\}$-hypoelliptic in $\Omega$ if $P u \in \mathcal{E}_{\{\omega\}}\left(\Omega^{\prime}\right)$ implies $u \in \mathcal{E}_{\{\omega\}}\left(\Omega^{\prime}\right)$ for every open set $\Omega^{\prime} \subset \Omega$ and for every $u \in \mathcal{D}_{\{\omega\}}^{\prime}\left(\Omega^{\prime}\right)$. 
We denote by $P_{x}$ (by $P_{y}$ respectively) the operator $P$ acting on the spaces $\mathcal{E}_{\{\omega\}}\left(\Omega_{x} \times \Omega_{y}\right)$ and $\mathcal{D}_{\{\omega\}}^{\prime}\left(\Omega_{x} \times \Omega_{y}\right)$ with respect to the $x$-variables (to the $y$ variables respectively). For example

$$
P_{x} \varphi(x, y)=\sum_{|\alpha| \leq m} a_{\alpha}(x) D_{x}^{\alpha} \varphi(x, y), \quad \varphi \in \mathcal{E}_{\{\omega\}}\left(\Omega_{x} \times \Omega_{y}\right),
$$

where $D_{x}^{\alpha}$ denotes the derivative of order $\alpha$ with respect to the $x$-variables. The operators $P_{x}: \mathcal{E}_{\{\omega\}}\left(\Omega_{x} \times \Omega_{y}\right) \rightarrow \mathcal{E}_{\{\omega\}}\left(\Omega_{x} \times \Omega_{y}\right)$ and $P_{x}: \mathcal{D}_{\{\omega\}}^{\prime}\left(\Omega_{x} \times \Omega_{y}\right) \rightarrow$ $\mathcal{D}_{\{\omega\}}^{\prime}\left(\Omega_{x} \times \Omega_{y}\right)$ correspond to the extended tensor products

$$
P \hat{\otimes} I: \mathcal{E}_{\{\omega\}}(\Omega) \hat{\otimes}_{\pi} \mathcal{E}_{\{\omega\}}(\Omega) \rightarrow \mathcal{E}_{\{\omega\}}(\Omega) \hat{\otimes}_{\pi} \mathcal{E}_{\{\omega\}}(\Omega)
$$

and

$$
P \hat{\otimes} I: \mathcal{D}_{\{\omega\}}^{\prime}(\Omega) \hat{\otimes}_{\pi} \mathcal{D}_{\{\omega\}}^{\prime}(\Omega) \rightarrow \mathcal{D}_{\{\omega\}}^{\prime}(\Omega) \hat{\otimes}_{\pi} \mathcal{D}_{\{\omega\}}^{\prime}(\Omega)
$$

via the canonical isomorphisms.

Definition 2.2. Let $\Omega \subset \mathbb{R}^{N}$ be an open set and let $K(x, y) \in \mathcal{D}_{\{\omega\}}^{\prime}\left(\Omega_{x}\right.$ $\left.\times \Omega_{y}\right)$. The kernel $K$ is said to be $\{\omega\}$-ultradifferentiable fundamental kernel of the differential operator $P_{x}$ if

$$
P_{x} K(x, y)-\delta(x-y)=0 .
$$

Here $\delta(x-y)$ denotes the Dirac measure on the diagonal $\Delta$ of $\Omega \times \Omega$ and corresponds to the canonical inclusion $i: \mathcal{D}_{\{\omega\}}(\Omega) \hookrightarrow \mathcal{D}_{\{\omega\}}^{\prime}(\Omega)$ via the isomorphism given in (2.5). The kernel $K$ is said to be a $\{\omega\}$-ultradifferentiable parametrix if $P_{x} K(x, y)-\delta(x-y) \in \mathcal{E}_{\{\omega\}}\left(\Omega_{x} \times \Omega_{y}\right)$. Finally, the kernel $K$ is said to be a two-sided $\{\omega\}$-ultradifferentiable kernel in $\Omega$ of the differential operator $P$ if

$$
P_{x} K(x, y)=\delta(x-y), \quad{ }^{t} P_{y} K(x, y)=\delta(x-y),
$$

or equivalently, if the corresponding map $K$ defined in (2.5) satifies

$$
K P \varphi=P K \varphi=\varphi
$$

for all $\varphi \in \mathcal{D}_{\{\omega\}}(\Omega)$.

The next result is due to Morando [20] for Gevrey classes.

Theorem 2.1. $\quad$ Let $P$ be a linear partial differential operator defined in an open set $\Omega \subset \mathbb{R}^{N}$ with coefficients in $\mathcal{E}_{\{\omega\}}(\Omega)$. If $P$ is $\{\omega\}$-hypoelliptic in $\Omega$, then every point of $\Omega$ has an open neighborhood in which ${ }^{t} P$ has a $\{\omega\}$ ultradifferentiable fundamental kernel. If ${ }^{t} P$ is also $\{\omega\}$-hypoelliptic in $\Omega$, then every point of $\Omega$ has an open neighborhood where $P$ has a two-sided $\{\omega\}$ ultradifferentiable fundamental kernel, which is $\{\omega\}$-very regular. 
We recall the following consequence of [2, Lemma 2.3 and Proof of Theorem 2.1].

Proposition 2.1. $\quad$ Let $P$ be a linear partial differential operator defined in an open set $\Omega \subset \mathbb{R}^{N}$ with coefficients in $\mathcal{E}_{\{\omega\}}(\Omega)$. Suppose that $P$ is $\{\omega\}$ hypoelliptic in $\Omega$. Then for every $x \in \Omega$ there is a compact subset $K \subset \Omega$ with $x \in \stackrel{\circ}{K}$ such that for every $\sigma \in S_{\omega}$ there exist $\sigma^{\prime} \in S_{\omega}, \sigma \leq \sigma^{\prime}$, and $c_{\sigma}>0$ for which

$$
\|\varphi\|_{2, \sigma} \leq c_{\sigma}\|P \varphi\|_{2, \sigma^{\prime}}
$$

for all $\varphi \in \mathcal{D}_{\{\omega\}}(K)$.

Remark. If ${ }^{t} P$ is also $\{\omega\}$-hypoelliptic in $\Omega$, for a fixed $x \in \Omega$ we can find a compact subset $K$ of $\Omega$ with $x \in \stackrel{\circ}{K}$ such that for every $\sigma \in S_{\omega}$ there exist $\sigma^{\prime} \in S_{\omega}, \sigma \leq \sigma^{\prime}$, and $c_{\sigma}>0$ for which inequalities (2.8) and

$$
\|\varphi\|_{2, \sigma} \leq c_{\sigma}\left\|^{t} P \varphi\right\|_{2, \sigma^{\prime}}, \quad \forall \varphi \in \mathcal{D}_{\{\omega\}}(K),
$$

are both satisfied.

The proof of Theorem 2.1 is inspired by the one of [24, Theorem 52.2] and relies on the projective description of the Roumieu classes given in $\S 1$.

Proof. Fix $x \in \Omega$. Since $P$ is $\{\omega\}$-hypoelliptic in $\Omega$, by Proposition 2.1 there exists a compact subset $K \subset \Omega$ with $x \in \stackrel{\circ}{K}$ such that for every $\sigma \in S_{\omega}$ there exist $\sigma^{\prime} \in S_{\omega}, \sigma \leq \sigma^{\prime}$, and $c_{\sigma}>0$ for which

$$
\|\varphi\|_{2, \sigma} \leq c_{\sigma}\|P \varphi\|_{2, \sigma^{\prime}}
$$

for all $\varphi \in \mathcal{D}_{\{\omega\}}(K)$. Then the operator $J:\left(\left\{P \psi: \psi \in \mathcal{D}_{\{\omega\}}(K)\right\},\|\|_{2, \sigma^{\prime}}\right) \rightarrow$ $\left(\mathcal{D}_{\{\omega\}}(K),\|\|_{2, \sigma}\right), P \varphi \rightarrow \varphi$, is well-defined, linear and continuous. Denoting by $M$ the closure of $P\left(\mathcal{D}_{\{\omega\}}(K)\right)$ in $H_{\{\omega\}}^{\sigma^{\prime}}(K), J$ can be extended as a continuous linear map $T_{0}$ from $M$ into $H_{\{\omega\}}^{\sigma}(K)\left(T_{0}(P \varphi)=\varphi\right.$ for all $\left.\varphi \in \mathcal{D}_{\{\omega\}}(K)\right)$. Let $p_{M}$ be the orthogonal projection of the Hilbert space $H_{\{\omega\}}^{\sigma^{\prime}}(K)$ onto $M$ and $T:=T_{0} \circ p_{M}$. Then $T: H_{\{\omega\}}^{\sigma^{\prime}}(K) \rightarrow H_{\{\omega\}}^{\sigma}(K)$ is a linear continuous operator such that $\left(T(\varphi)=T_{0}(\varphi)\right.$ for all $\left.\varphi \in M\right)$

$$
T(P \varphi)=\varphi
$$

for all $\varphi \in \mathcal{D}_{\{\omega\}}(K)$. Next, we consider its transposed map ${ }^{t} T:\left(H_{\{\omega\}}^{\sigma}(K)\right)^{\prime} \rightarrow$ $\left(H_{\{\omega\}}^{\sigma^{\prime}}(K)\right)^{\prime}$. Since $\mathcal{D}_{\{\omega\}}(K)$ and $\left(H_{\{\omega\}}^{\sigma^{\prime}}(K)\right)^{\prime}$ are continuously embedded in 
$\left(H_{\{\omega\}}^{\sigma}(K)\right)^{\prime}$ and $\mathcal{D}_{\{\omega\}}^{\prime}(K)$ respectively (see $\left.\S 1\right)$, we have

$$
\mathcal{D}_{\{\omega\}}(K) \hookrightarrow\left(H_{\{\omega\}}^{\sigma}(K)\right)^{\prime} \stackrel{t_{T}}{\rightarrow}\left(H_{\{\omega\}}^{\sigma^{\prime}}(K)\right)^{\prime} \hookrightarrow \mathcal{D}_{\{\omega\}}^{\prime}(K),
$$

i.e. we can regard ${ }^{t} T \in L_{\beta}\left(\mathcal{D}_{\{\omega\}}(K), \mathcal{D}_{\{\omega\}}^{\prime}(K)\right) \simeq \mathcal{D}_{\{\omega\}}^{\prime}(K \times K)$ (see [12, Theorem 2.1], [13, §5]). Moreover, from $(2.11)$ we obtain that ${ }^{t} P\left({ }^{t} T \varphi\right)=\varphi$ for all $\varphi \in \mathcal{D}_{\{\omega\}}(K)$. Thus, the kernel associated with ${ }^{t} T$ is a $\{\omega\}$-fundamental kernel of ${ }^{t} P$ in $K$, hence in the open neighbourhood $U=\stackrel{\circ}{K}$ of $x$. Thus, the first statement of the theorem is proved.

Assume that ${ }^{t} P$ is $\{\omega\}$-hypoelliptic in $\Omega$ too. By Proposition 2.1 and Remark thereafter we can proceed as before to obtain a continuous linear map $S: H_{\{\omega\}}^{\sigma^{\prime}}(K) \rightarrow H_{\{\omega\}}^{\sigma}(K)$ such that $S\left({ }^{t} P \varphi\right)=\varphi$ for all $\varphi \in \mathcal{D}_{\{\omega\}}(K)$; hence, for all $u \in \mathcal{D}_{\{\omega\}}^{\prime}(K)$

$$
P\left({ }^{t} S u\right)=u \text {. }
$$

Since the spaces $H_{\{\omega\}}^{\sigma^{\prime}}(K)$ and $L^{2}\left(\mathbb{R}^{N}\right)$ are continuously included in $L^{2}\left(\mathbb{R}^{N}\right)$ and $\left(H_{\{\omega\}}^{\sigma}(K)\right)^{\prime}$ respectively (see $\left.(1.2)\right)$, we can define the map

$$
E:=T p_{M}+{ }^{t} S\left(I-p_{M}\right),
$$

here $I$ denotes the identity map of $H_{\{\omega\}}^{\sigma^{\prime}}(K)$. Then $E \in L_{\beta}\left(\mathcal{D}_{\{\omega\}}(K), \mathcal{D}_{\{\omega\}}^{\prime}(K)\right)$. By (2.11) and (2.12) we can proceed as in [24, Theorem 52.2] to show that $E(P \varphi)=\varphi$ and $P(E \varphi)=\varphi$ for all $\varphi \in \mathcal{D}_{\{\omega\}}(K)$. Consequently the kernel in $\mathcal{D}_{\{\omega\}}^{\prime}(K \times K)$ corresponding to $E$ is a two-sided $\{\omega\}$-ultradifferentiable fundamental kernel of $P$ in $K$, hence in $U$. It remains to show that $E$ is $\{\omega\}$-very regular. This is a consequence of the following lemma.

Lemma 2.1. $\quad$ Let $P$ be a linear partial differential operator defined in an open set $\Omega \subset \mathbb{R}^{N}$ with coefficients in $\mathcal{E}_{\{\omega\}}(\Omega)$. If $P$ and ${ }^{t} P$ are both $\{\omega\}$ hypoelliptic in $\Omega$, then every two-sided $\{\omega\}$-ultradifferentiable fundamental kernel of $P$ in $\Omega$ is $\{\omega\}$-very regular.

Proof. Let $K \in \mathcal{D}_{\{\omega\}}^{\prime}(\Omega \times \Omega)$ be a two-sided $\{\omega\}$-ultradifferentiable fundamental kernel of $P$ in $\Omega$. Proceeding as in [24, Lemma 52.1] and using the closed graph theorem for webbed spaces (see e.g. [10, Theorem 5.4.1, Proposition 5.2.2 and Corollary 5.3.3]), we obtain that the kernel $K$ is $\{\omega\}$-regular. It remains to show that the kernel $K$ is $\{\omega\}$-very regular.

Since the following isomorphisms of locally convex spaces

$$
L_{\beta}\left(\mathcal{D}_{\{\omega\}}(\Omega), \mathcal{E}_{\{\omega\}}(\Omega)\right) \simeq \mathcal{E}_{\{\omega\}}\left(\Omega, \mathcal{D}_{\{\omega\}}^{\prime}(\Omega)\right) \simeq \mathcal{D}_{\{\omega\}}^{\prime}(\Omega) \hat{\otimes}_{\pi} \mathcal{E}_{\{\omega\}}(\Omega)
$$


hold (see e.g. [13, Theorem 5.2]), $K(x, y) \in \mathcal{E}_{\{\omega\}}\left(\Omega, \mathcal{D}_{\{\omega\}}^{\prime}(\Omega)\right.$ ), and we can proceed as in [24, Lemma 52.1] again to show that, given two open subsets $U$ and $V$ of $\Omega$ such that $U \cap V=\emptyset$, the set $\mathcal{H}:=\left\{\partial_{y}^{\alpha} K(x, y): \alpha \in \mathbb{Z}_{+}^{n}, y \in V\right\} \subset$ $\mathcal{D}_{\{\omega\}}^{\prime}\left(U_{x}\right)$ is contained in $\operatorname{Ker} P=\left\{u \in \mathcal{D}_{\{\omega\}}^{\prime}\left(U_{x}\right): P u=0\right\} \subset \mathcal{E}_{\{\omega\}}\left(U_{x}\right)$, where Ker $P$ is clearly a closed subspace both of $\mathcal{D}_{\{\omega\}}^{\prime}\left(U_{x}\right)$ and of $\mathcal{E}_{\{\omega\}}\left(U_{x}\right)$. Thus, Ker $P$ is a Fréchet space with respect to the topology $\tau_{2}$ induced by $\mathcal{D}_{\{\omega\}}^{\prime}\left(U_{x}\right)$ and is a webbed space with respect to the topology $\tau_{1}$ induced by $\mathcal{E}_{\{\omega\}}\left(U_{x}\right)$ (see e.g. [10, Theorem 5.3.1]). Since the inclusion map $i:\left(\operatorname{Ker} P, \tau_{1}\right) \hookrightarrow\left(\operatorname{Ker} P, \tau_{2}\right)$ is continuous, $i$ is then a topological isomorphism by the open mapping theorem for webbed spaces (see e.g. [10, Theorem 5.5.2]). Since $\mathcal{E}_{\{\omega\}}\left(U_{x}\right)$ and $\mathcal{D}_{\{\omega\}}^{\prime}\left(U_{x}\right)$ induce the same topology on $\operatorname{Ker} P$ and hence on $\mathcal{H}$, we conclude $K(x, y) \in$ $\mathcal{E}_{\{\omega\}}\left(V_{y}, \mathcal{E}_{\{\omega\}}\left(U_{x}\right)\right) \simeq \mathcal{E}_{\{\omega\}}\left(V_{y} \times U_{x}\right)$. Indeed, let $\left(q_{l}\right)_{l}$ be a fundamental sequence of continuous seminorms on $\mathcal{D}_{\{\omega\}}^{\prime}\left(U_{x}\right)$. As $K(x, y) \in \mathcal{E}_{\{\omega\}}\left(V_{y}, \mathcal{D}^{\prime}\left(U_{x}\right)\right)$, for each compact subset $H$ of $V$ and each $l \in \mathbb{N}$, there is $m \in \mathbb{N}$ so that

$$
\sup _{y \in H} \sup _{\alpha \in \mathbb{Z}_{+}^{n}} q_{l}\left(\partial_{y}^{\alpha} K(., y)\right) \exp \left(-m \varphi^{*}\left(\frac{|\alpha|}{m}\right)\right)<+\infty .
$$

On the other hand, denoting by $\left(p_{i}\right)_{i \in I}$ a fundamental system of continuous seminorms on $\mathcal{E}_{\{\omega\}}\left(U_{x}\right)$, we have that

$$
\forall i \in I \exists l(i) \in \mathbb{N}, c_{i}>0 \forall f \in \operatorname{Ker} P \quad p_{i}(f) \leq c_{i} q_{l(i)} .
$$

Consequently for each compact subset $H$ of $V$ and $i \in I$ there is $m \in \mathbb{N}$ such that

$$
\sup _{y \in H} \sup _{\alpha \in \mathbb{Z}_{+}^{n}} p_{i}\left(\partial_{y}^{\alpha} K(., y)\right) \exp \left(-m \varphi^{*}\left(\frac{|\alpha|}{m}\right)\right)<+\infty .
$$

Thus $K(x, y) \in \mathcal{E}_{\{\omega\}}\left(V_{y}, \mathcal{E}\left(U_{x}\right)\right) \simeq \mathcal{E}_{\{\omega\}}\left(V_{y} \times U_{x}\right)$. This completes the proof.

Proposition 2.2. $\quad$ Let $P$ be a linear partial differential operator defined in an open set $\Omega \subset \mathbb{R}^{N}$ with coefficients in $\mathcal{E}_{\{\omega\}}(\Omega)$. Suppose that both $P$ and ${ }^{t} P$ are $\{\omega\}$-hypoelliptic in $\Omega$ and map $\mathcal{E}_{\{\omega\}}(\Omega)$ onto itself. Let $F$ be a complete locally convex space. Consider the following conditions:

(a) $P \hat{\otimes} I: \mathcal{E}_{\{\omega\}}(\Omega) \hat{\otimes}_{\pi} F \rightarrow \mathcal{E}_{\{\omega\}}(\Omega) \hat{\otimes}_{\pi} F$ is surjective.

(b) $P \hat{\otimes} I: \mathcal{D}_{\{\omega\}}^{\prime}(\Omega) \hat{\otimes}_{\pi} F \rightarrow \mathcal{D}_{\{\omega\}}^{\prime}(\Omega) \hat{\otimes}_{\pi} F$ is surjective.

Then (a) implies (b), and (b) implies (a) if $F_{\tau}^{\prime}$ is ultrabornological; for example if $F$ is a reflexive Fréchet or $(D F)$ space.

Proof. Let $F$ be a given complete locally convex space. 
(a) $\Longrightarrow$ (b) Let $S \in \mathcal{D}_{\{\omega\}}^{\prime}(\Omega) \hat{\otimes}_{\pi} F$. Since $P$ and ${ }^{t} P$ are both $\{\omega\}$-hypoelliptic in $\Omega$ and the spaces $\mathcal{E}_{\{\omega\}}(V)$ and $\mathcal{D}_{\{\omega\}}^{\prime}(V)$ induce the same topology on $N(\omega$, $P, V)$ for all open subset $V$ of $\Omega$ (see proof of Lemma 2.1), we can apply Theorem 2.1 and proceed as in [24, Theorem 52.3] to construct an element $T \in \mathcal{E}_{\{\omega\}}(\Omega) \hat{\otimes}_{\pi} F$ such that $S-(P \hat{\otimes} I)(T) \in \mathcal{E}_{\{\omega\}}(\Omega) \hat{\otimes}_{\pi} F$. By assumption there is then an element $R \in \mathcal{E}_{\{\omega\}}(\Omega) \hat{\otimes}_{\pi} F$ such that $(P \hat{\otimes} I) R=S-(P \hat{\otimes} I)(T)$, thereby implying that

$$
(P \hat{\otimes} I)(T+R)=S,
$$

and (b) follows.

(b) $\Longrightarrow\left(\right.$ a) if $F_{\tau}^{\prime}$ is ultrabornological. Fix $v \in \mathcal{E}_{\{\omega\}}(\Omega) \hat{\otimes}_{\pi} F$, by (b) there is $u \in \mathcal{D}_{\{\omega\}}^{\prime}(\Omega) \hat{\otimes}_{\pi} F$ such that $(P \hat{\otimes} I) u=v$. By the canonical isomorphism (1.5) $u$ defines a continuous linear map from $\mathcal{D}_{\{\omega\}}(\Omega)$ into $F$, which we denote again by $u$, such that

$$
f \circ u \in \mathcal{D}_{\{\omega\}}^{\prime}(\Omega) \text { and } P(f \circ u)=f \circ(P \hat{\otimes} I) u=f \circ v \in \mathcal{E}_{\{\omega\}}(\Omega)
$$

for all $f \in F^{\prime}$. Since $P$ is $\{\omega\}$-hypoelliptic in $\Omega$, by (2.13) we get $f \circ u \in \mathcal{E}_{\{\omega\}}(\Omega)$ for all $f \in F^{\prime}$. Therefore $u: \mathcal{D}_{\{\omega\}}(\Omega) \rightarrow F$ is $\sigma\left(\mathcal{D}_{\{\omega\}}(\Omega), \mathcal{E}_{\{\omega\}}(\Omega)\right)-\sigma\left(F^{\prime}, F\right)$ continuous, hence $u^{t}: F_{\tau}^{\prime} \rightarrow \mathcal{E}_{\{\omega\}}(\Omega)$ is well defined and continuous if we endow $\mathcal{E}_{\{\omega\}}(\Omega)$ with the topology induced by $\mathcal{D}_{\{\omega\}}^{\prime}(\Omega)$, therefore $u^{t}: F_{\tau}^{\prime} \rightarrow \mathcal{E}_{\{\omega\}}(\Omega)$ has closed graph. Since $F_{\tau}^{\prime}$ is ultrabornological, we can apply the closed graph theorem for webbed spaces (see e.g. [10, Theorem 5.4.1, Proposition 5.2.2 and Corollary 5.3.3]) to conclude that $u^{t}$ is continuous. This yields $u \in \mathcal{E}_{\{\omega\}}(\Omega, F)$ and (a) follows by (1.4).

As immediate consequence of Proposition 2.2 we obtain the following.

Corollary 2.1. $\quad$ Let $P$ be a linear partial differential operator defined in an open set $\Omega \subset \mathbb{R}^{N}$ with coefficients in $\mathcal{E}_{\{\omega\}}(\Omega)$. Suppose that both $P$ and ${ }^{t} P$ are $\{\omega\}$-hypoelliptic in $\Omega$ and map $\mathcal{E}_{\{\omega\}}(\Omega)$ onto itself. Then the following holds.

(1) The operator $P: \mathcal{D}_{\{\omega\}}^{\prime}(\Omega) \rightarrow \mathcal{D}_{\{\omega\}}^{\prime}(\Omega)$ is surjective.

(2) The operators $P \hat{\otimes} I$ and ${ }^{t} P \hat{\otimes} I$ are surjective on the spaces $\mathcal{E}_{\{\omega\}}(\Omega) \hat{\otimes}_{\pi} F$ and $\mathcal{D}_{\{\omega\}}^{\prime}(\Omega) \hat{\otimes}_{\pi} F$ for every reflexive Fréchet space $F$.

Proof. In order to show (1), we have only to apply Proposition 2.2, (a) $\Longrightarrow($ b) with $F=\mathbb{C}$. To show (2), let $F$ be a reflexive Fréchet space. Since $\mathcal{D}_{\{\omega\}}^{\prime}(\Omega)$ is a Fréchet space and $P$ is surjective, a well-known theorem 
of Grothendieck [6] implies that $P \hat{\otimes} I: \mathcal{D}_{\{\omega\}}^{\prime}(\Omega) \hat{\otimes}_{\pi} F \rightarrow \mathcal{D}_{\{\omega\}}^{\prime}(\Omega) \hat{\otimes}_{\pi} F$ is surjective, hence $P \hat{\otimes} I: \mathcal{E}_{\{\omega\}}(\Omega) \hat{\otimes}_{\pi} F \rightarrow \mathcal{E}_{\{\omega\}}(\Omega) \hat{\otimes}_{\pi} F$ is surjective too by Proposition $2.2,(\mathrm{~b}) \Longrightarrow(\mathrm{a})$. Similarly, one shows the result for ${ }^{t} P \hat{\otimes} I$.

We note that the preceding proofs rely heavily on the $\{\omega\}$-hypoellipticity of $P$ and of ${ }^{t} P$ to establish that the surjectivity of $P$ on the function space $\mathcal{E}_{\{\omega\}}(\Omega)$ implies the surjectivity of $P \hat{\otimes}_{\pi} I$ on the vector valued spaces $\mathcal{E}_{\{\omega\}}(\Omega) \hat{\otimes}_{\pi} F$ and $\mathcal{D}_{\{\omega\}}^{\prime}(\Omega) \hat{\otimes}_{\pi} F$.

The following theorem extends a well-known result of Malgrange [17] (see also [24, Theorem 52.3]) to the setting of non-quasianalytic classes of Roumieu type.

Theorem 2.2. $\quad$ Let $P$ be a linear partial differential operator defined in an open set $\Omega \subset \mathbb{R}^{N}$ with coefficients in $\mathcal{E}_{\{\omega\}}(\Omega)$. Suppose that both $P$ and ${ }^{t} P$ are $\{\omega\}$-hypoelliptic in $\Omega$ and map $\mathcal{E}_{\{\omega\}}(\Omega)$ onto itself. Then $P$ has a two-sided $\{\omega\}$-ultradifferentiable fundamental kernel in $\Omega$ which is $\{\omega\}$-very regular.

Proof. By Corollary $2.1(2)$, with $F=\mathcal{D}_{\{\omega\}}^{\prime}(\Omega)$, there exists

$$
K_{1} \in \mathcal{D}_{\{\omega\}}^{\prime}\left(\Omega_{x}\right) \hat{\otimes} \mathcal{D}_{\{\omega\}}^{\prime}\left(\Omega_{y}\right) \text { with }(P \hat{\otimes} I) K_{1}(x, y)=I_{x, y} .
$$

Here $I_{x}, I_{y}$ denote the identity maps in the spaces of $\{\omega\}$-ultradistributions in the variables $x, y$ and $I_{x, y}$ denotes the canonical inclusion $i: \mathcal{D}_{\{\omega\}}(\Omega) \hookrightarrow$ $\mathcal{D}_{\{\omega\}}^{\prime}(\Omega)$ respectively. Proceeding as at the end of [24, Theorem 52.3] we put $L:=I_{x, y}-\left(I_{x} \hat{\otimes}^{t} P_{y}\right) K_{1} \in \mathcal{D}_{\{\omega\}}^{\prime}\left(\Omega_{x}\right) \hat{\otimes}_{\pi} \mathcal{D}_{\{\omega\}}^{\prime}\left(\Omega_{y}\right)$, thereby obtaining that $L \in$ $L\left(\mathcal{D}_{\{\omega\}}\left(\Omega_{y}\right)\right.$, Ker $\left.P\right)$, when Ker $P$ is endowed with the Fréchet-topology induced by $\mathcal{D}_{\{\omega\}}^{\prime}\left(\Omega_{x}\right)$. Taking $F=\operatorname{Ker} P$ and ${ }^{t} P_{y}$ instead of $P$, we apply again Corollary 2.1 (2), to obtain $K_{2}(x, y) \in \operatorname{Ker} P \hat{\otimes}_{\pi} \mathcal{D}_{\{\omega\}}^{\prime}\left(\Omega_{y}\right)$ (hence $\left.\left(P_{x} \hat{\otimes} I_{y}\right) K_{2}=0\right)$ such that $\left(I_{x} \hat{\otimes}^{t} P_{y}\right) K_{2}=L$. Putting $K=K_{1}+K_{2}$, it follows that $\left(I_{x} \hat{\otimes}^{t} P_{y}\right) K=I_{x, y}$ and $\left(P_{x} \hat{\otimes} I_{y}\right) K=I_{x, y}$. By the canonical isomorphism (2.2) this means that $K$ is a two-sided $\{\omega\}$-ultradifferentiable fundamental kernel of $P$ in $\Omega$. By Lemma $2.1 K$ is also $\{\omega\}$-very regular and the proof is complete.

The following consequence is also new for Gevrey classes.

Corollary 2.2. $\quad$ Let $P$ be a linear partial differential operator defined in an open set $\Omega \subset \mathbb{R}^{N}$ whose coefficients belong to $G^{s}(\Omega)$ with $s>1$. Suppose that both $P$ and ${ }^{t} P$ are s-hypoelliptic in $\Omega$ and map $G^{s}(\Omega)$ onto itself. Then $P$ has a two-sided s-ultradifferentiable fundamental kernel in $\Omega$ which is s-very regular. 
Example 2. Consider the elliptic partial differential operator of order $2 m$

$$
P=P(x, D)=\sum_{|p|,|q| \leq m}(-1)^{|p|} D^{p}\left(a_{p q}(x) D^{q}\right), \quad x \in \mathbb{R}^{N} .
$$

Suppose that the following conditions are satisfied: all the coefficients $a_{p q}$ are real-valued and belong to $G^{s}\left(\mathbb{R}^{N}\right), s>1$, and the characteristic form $P_{0}(x, \xi)$ of the operator $P$ satisfies

$$
P_{0}(x, \xi)=\sum_{|p|,|q|=m}(-1)^{m} a_{p q}(x) \xi^{p+q} \geq \mu(x)|\xi|^{2 m}, \quad x, \xi \in \mathbb{R}^{N},
$$

where $\inf _{K} \mu>0$ for every compact subset $K$ of $\mathbb{R}^{N}$.

Under these hypothesis, we have that for every $g \in G^{s}\left(\mathbb{R}^{N}\right)$ and $n \in \mathbb{N}$ there is $u_{n} \in G^{s}\left(B_{n}\right) \cap C\left(\overline{B_{n}}\right)\left(B_{n}:=\left\{x \in \mathbb{R}^{N}:|x|<n\right\}\right)$ satisfying the following Dirichlet problem

$$
\begin{cases}P u_{n}=g & \text { on } B_{n} \\ \frac{\partial^{j} u_{n}}{\partial \nu^{j}}=0, j=0, \ldots, m-1, & \text { on } \partial B_{n}\end{cases}
$$

where $\nu$ denotes the normal vector field to $\partial B_{n}$, oriented towards the interior of $B_{n}$, see [16, Ch. 8, Theorem 1.1 and Corollary 1.1] and [15, Ch. 2, §1]. Thus $P$ is locally surjective in $G^{s}\left(\mathbb{R}^{N}\right)$ in the sense of Braun, Meise and Vogt [4], i.e. for every $g \in G^{s}\left(\mathbb{R}^{N}\right)$ and $n \in \mathbb{N}$ there exists $f \in G^{s}\left(\mathbb{R}^{N}\right)$ such that $P(f)_{\mid B_{n}}=g_{\mid B_{n}}$. This corresponds to the concept of semiglobally solvable operator in the sense of Trèves [24, Definition 38.2, p. 392].

Indeed, if we put $f:=\chi_{n+1} u_{n+1}$ where $u_{n+1}$ is the solution of the Dirichlet problem on $B_{n+1}$ associated to $P$ (here $\chi_{n+1} \in G_{0}^{s}\left(\mathbb{R}^{N}\right)$ satisfies $\chi_{n+1} \equiv 1$ on $B_{n}, 0 \leq \chi_{n+1} \leq 1$, and $\left.\operatorname{supp} \chi_{n+1} \subset B_{n+1}\right)$, we have that $f \in G^{s}\left(\mathbb{R}^{N}\right)$ and $P(f)_{\mid B_{n}}=P\left(u_{n+1}\right)_{\mid B_{n}}=g_{\mid B_{n}}$.

Since Ker $P$ is a nuclear Fréchet space (see proof of Lemma 2.1), we can apply [1, Proposition 3.1] to obtain that $P$ is a surjective map on $G^{s}\left(\mathbb{R}^{N}\right)$. As it is easy to verify, ${ }^{t} P$ also satisfies all the above conditions and then ${ }^{t} P$ is a surjective map on $G^{s}\left(\mathbb{R}^{N}\right)$. As $P$ and ${ }^{t} P$ are elliptic partial differential operators in $\mathbb{R}^{N}$, hence $s$-hypoelliptic in $\mathbb{R}^{N}$, by Corollary $2.2 P$ has a twosided $s$-ultradifferentiable fundamental kernel in $\mathbb{R}^{N}$ which is $s$-very regular.

We refer the reader to the article [1] for more examples and related results. 


\section{Acknowledgement}

The authors thank C. Fernández and A. Galbis for helpful suggestions on the subject of this article.

\section{References}

[1] A. A. Albanese, Surjective linear partial differential operators with variable coefficients on non-quasianalytic classes of Roumieu type, in Hyperbolic Problems and Regularity Questions, Trends in Math., Birkhäuser Verlag, Basel 2006, pp. 7-16.

[2] A. A. Albanese, A. Corli and L. Rodino, Hypoellipticity and local solvability in Gevrey classes, Math. Nachr. 242 (2002), 5-16.

[3] R. W. Braun, R. Meise and B. A. Taylor, Ultradifferentiable functions and Fourier analysis, Results Math. 17 (1990), no. 3-4, 206-237.

[4] R. W. Braun, R. Meise and D. Vogt, Characterization of the linear partial differential operators with constant coefficients which are surjective on nonquasianalytic classes of Roumieu type on $\mathbb{R}^{N}$, Math. Nachr. 168 (1994), 19-54.

[5] L. Ehrenpreis, Solution of some problems of division. I. Division by a polynomial of derivation, Amer. J. Math. 76 (1954), 883-903.

[6] A. Grothendieck, Produits tensoriels topologiques et espaces nucléaires, Mem. Amer. Math. Soc. 16, Providence 1955.

[7] L. Hörmander, Pseudo-differential operators and non-elliptic boundary problems, Ann. of Math. (2) 83 (1966), 129-209.

[8] - Linear partial differential operators, Springer, Berlin, 1976.

[9] - The Analysis of Linear Partial Differential Operators, I-IV, Springer-Verlag, Berlin Heidelberg New York, 1983.

[10] H. Jarchow, Locally convex spaces, Teubner, Stuttgart, 1981.

[11] H. Komatsu, Ultradistributions. I. Structure theorems and a characterization, J. Fac. Sci. Univ. Tokyo Sect. IA Math. 20 (1973), 25-105.

[12] Ultradistributions. II. The kernel theorem and ultradistributions with support in a submanifold, J. Fac. Sci. Univ. Tokyo Sect. IA Math. 24 (1977), no. 3, 607-628.

[13] Ultradistributions. III. Vector-valued ultradistributions and the theory of kernels, J. Fac. Sci. Univ. Tokyo Sect. IA Math. 29 (1982), no. 3, 653-717.

[14] G. Köthe, Topological vector spaces. II, Springer, New York, 1979.

[15] J. L. Lions and E. Magenes, Non-Homogeneous Boundary Value Problems and Applications, I, Springer-Verlag, Berlin, 1972.

[16] _ Non-homogeneous boundary value problems and applications. Vol. III, Translated from the French by P. Kenneth, Springer, New York, 1973.

[17] B. Malgrange, Existence et approximation des solutions des équations aux dérivées partielles et des équations de convolution, Ann. Inst. Fourier, Grenoble 6 (1955-1956), 271-355.

[18] R. Meise and B. A. Taylor, Whitney's extension theorem for ultradifferentiable functions of Beurling type, Ark. Mat. 26 (1988), no. 2, 265-287.

[19] R. Meise, B. A. Taylor and D. Vogt, Continuous linear right inverses for partial differential operators on non-quasianalytic classes and on ultradistributions, Math. Nachr. 180 (1996), 213-242.

[20] A. Morando, Hypoellipticity and local solvability of pseudolocal continuous linear operators in Gevrey classes, Tsukuba J. Math. 28 (2004), no. 1, 137-153.

[21] L. Rodino, Linear partial differential operators in Gevrey spaces, World Sci. Publishing, River Edge, NJ, 1993.

[22] L. Schwartz, Espaces de fonctions différentiables à valeurs vectorielles, J. Analyse Math. $4(1954 / 55), 88-148$. 
[23] L. Schwartz, Théorie des distributions à valeurs vectorielles. I, II, Ann. Inst. Fourier, Grenoble 7 (1957), 1-141; ibid. 8 (1958), 1-209.

[24] F. Trèves, Topological vector spaces, distributions and kernels, Academic Press, New York, 1967.

[25] D. Vogt, Topics on projective spectra of (LB)-spaces, in Advances in the theory of Fréchet spaces (Istanbul, 1988), 11-27, Kluwer Acad. Publ., Dordrecht.

[26] J. Wengenroth, Derived functors in functional analysis, Lecture Notes in Math., 1810, Springer, Berlin, 2003. 